\title{
Los indios americanos en las escenas líricas europeas: de los hermanos Purcell (1695) a Carl Heinrich Graun (1755)
}

Por

\author{
Ana Núñez Ronchi \\ Université de Lorraine
}

Se ha querido interpretar las primeras óperas de tema americano (Purcell, Vivaldi, Rameau y Graun) como una expresión dramático-musical de la llamada Leyenda Negra, contrapuesta a una producción teatral áurea española que, inversamente, si habría dejado espacio a la dimensión histórico-crítica. Ello nos parece injusto e inexacto: en primer lugar, la comedia áurea no escenifica la dimensión crítica que se ha pretendido atribuirle; en segundo lugar, el teatro lírico europeo, si bien exalta al indio americano, no devalúa sistemáticamente a los conquistadores españoles; finalmente, la noción misma de «Leyenda Negra» debe ser problematizada.

Palabras ClaVE: ópera; indios americanos; Leyenda Negra; semántica interpretativa.

A pesar (o tal vez a causa) de la frenética actividad exploradora y colonizadora que desplegó la Corona española en América, tenemos constancia de que las primeras óperas de la historia de la música española, indiferentes a las posibilidades líricas que ofrecía una humanidad recién descubierta, persistían en mantener vigentes los grandes temas de la mitología occidental ${ }^{1}$. Mientras tanto, en el espacio de algo más de medio siglo, los grandes escenarios europeos se encendían con la presencia de cuatro piezas operísticas de tema

1 Óperas enteramente cantadas o géneros mixtos como zarzuelas o semióperas tales como La selva sin amor de Lope de Vega (1527), La fiera, el rayo y la piedra (1652), Fortuna de Andromeda y Perseo (1653), El golfo de las sirenas (1657), El laurel de Apolo (1658), Celos aun del aire matan (1660?), La púrpura de la rosa (1660), Fieras afemina amor (1670 o 1672) o La estatua de Prometeo (ha. 1670-75), con libreto de Calderón de la Barca, descuidan todas el tema americano y se centran en la mitología clásica. 
americano: la semi-ópera The Indian Queen de Henry y Daniel Purcell (Theater Royal, Drury Lane, 1696) ${ }^{2}$, con libreto de autor controvertido ${ }^{3}$, Motezuma $^{4}$ de Antonio Vivaldi (Venecia, Teatro S. Angelo, 14-11-1733), con libreto de Alvise Giusti, Les Indes Galantes de Jean-Philippe Rameau (París, Les Tuileries, 23-08-1735), con libreto de Louis Fuzelier, y Montezuma de Carl Heinrich Graun (Berlín, Königlisches Opernhaus, 6-01-1755), con libreto en francés de Federico II el Grande -de quien precisamente en 2012 se celebra el 300 aniversario de su nacimiento- traducido al italiano por Giampietro Tagliazucchi.

Se ha querido ver en estas piezas, raramente tratadas conjuntamente, una expresión dramática y/o musical de la llamada Leyenda Negra ${ }^{5}$, supuestamente contrapuesta a una producción teatral áurea española que, inversamente, sí habría dejado espacio a la dimensión crítica ${ }^{6}$. Ello nos parece injusto e inexacto, por varias razones; en primer lugar, porque la comedia del Siglo de Oro no escenifica la dimensión crítica que se ha pretendido atribuirle ${ }^{7}$; en segundo lugar, porque el teatro lírico europeo, si bien exalta al indio americano, no devalúa sistemáticamente a los conquistadores españoles; finalmen-

2 La primera representación fechada se remonta al 29 de abril de 1696, cinco meses después de la muerte de Henry Purcell, aunque algunos sostienen que tuvo lugar antes del 21 de noviembre de 1695, fecha de la muerte de compositor (Asch, 1995: 2). Price (1984: 126), uno de los mayores especialistas en la materia, propone los últimos meses de la temporada de otoño de 1695 como fecha más probable. Gildon se refiere al «General Applause» (1699: 75) que suscitó el estreno de la semiópera. Subsiguientemente, reapareció en las carteleras londinenses en numerosas ocasiones: abril de 1696, marzo, junio y diciembre de 1697, enero de 1701, abril de 1706 y julio de 1715 (Dryden, 1956-2002a: 325-326). En 1998, la compañía de ópera de Long Beach volvía a presentar, en una producción chicana de tintes paródicos, una versión operística de La reina india (Swed, 1998).

3 Al igual que con la pieza teatral homónima, la contribución en la adaptación del libreto por parte de John Dryden ha sido muy debatida, barajándose también la posibilidad de una adaptación por parte de Robert Howard o del actor Thomas Betterton. Dada la estrecha colaboración entre Purcell y el matrimonio Howard, las conclusiones de la Purcell Society apuntan más bien hacia este último (Pinnock y Laurie, 1994: escena xi), Price (1984: 126-127) sostiene, por el contrario, que Dryden, leal colaborador de la compañía, pudo haber hecho el trabajo que Betterton, enemistado con los empresarios del Theater Royal, se rehusaba a emprender.

${ }^{4}$ Conservamos la grafía de los textos originales.

5 Orr, 2001: 143. Moreno Mengíbar, 2012. Duviols, 2006: 318. Fantoni, 192.

6 «[las indígenas son] voces difícilmente audibles en otras colectividades colonizadoras de entonces o de más tarde, las cuales cuando colonizaron no las dejaron hablar en voz alta en los escenarios, públicos o privados, de la conciencia histórica colectiva» (Ruiz Ramón, 1993: 13).

7 Para el caso del teatro áureo, véase Núñez Ronchi, 2013: 389-414. 
te, porque la noción misma de «Leyenda Negra», no obstante su éxito y su persistencia, debe ser problematizada. En la perspectiva de una semiótica de las culturas, que es la que propugna el lingüista francés François Rastier, y que adoptamos aquí, es importante precisar que:

Loin de refléter la réalité, les signes la créent, et prenant cet effet (ou ce corrélat) pour une cause nous nommons réalité cet effet de réel. Toute philosophie a certes une fonction réaliste, comme le notait Bachelard. Mais n'est-il pas vrai que toute la philosophie occidentale s'organise autour de la question de l'Être?

Se hace además imprescindible mencionar aquí la particularidad del campo genérico lírico-teatral desde el punto de vista del componente dialógico, que es el que da cuenta de las modalidades veridictorias (factuales, contrafactuales, posibles) de las unidades textuales (Rastier, 2001: 39-41). En efecto, si en el relato clásico los enunciados son asumidos por un narrador único que permite el encuadramiento relativamente homogéneo de los enunciados, no sucede lo mismo en los textos de tipo dramático, donde la multiplicidad de los enunciadores apunta hacia la multiplicidad de modalidades veridictorias o evaluativas. Así, en el teatro, «las indicaciones escénicas, y a veces las palabras de un recitante, aseguran la función de narración sin que esté presente el narrador como actor del relato; fuera de ésto, queda asegurada por los actores del relato mismo. [...] Un enunciado será verdadero si puede ser confirmado por un actante oponente» (Rastier, 1973: 93-94)9.

8 Rastier, 1992: 120. En efecto, «les objets sont constitués par des évaluations sociales, et non par des données physiques» (Rastier, 1997). «Le positivisme consisterait [...] à éluder ainsi l'interprétation et à présenter comme des faits scientifiques de simples données qui ne seraient pourtant véritablement établies comme telles que par leur interprétation» (Rastier, 2010).

9 Algunos enunciados pueden ser objeto, además, de inferencias que modifiquen su valor de verdad. Tal es, por ejemplo, el caso de la ironía o de las aferencias comúnmente adscritas a un actor determinado: así, el Demonio puede recibir, por aferencia, el rasgo /mentiroso/, siempre y cuando el texto considerado actualice ese sema: tal es el caso en Jn. 9, 44 en la Biblia, en Acosta: 403, lib. VII, cap. 23) y en Fernando de Zárate: «RELIGIÓN: Siempre menos me dirás, / porque siempre has de mentir. / Del padre de la mentira / eres hija» (1668: 242). En otros casos, permanecen virtualizadas por instrucción contextual: «También se ha de entender que aunque el demonio es padre de la mentira, pero a su pesar le hace el rey de gloria, confesar la verdad muchas veces, y aun él mismo de puro miedo y despecho la dice no pocas. Asi daba voces en el desierto» (Acosta, 1940: 360, lib. VII, cap. 3). Estas aferencias pueden venir constreñidas por el discurso y el género textual. 
La semiópera The Indian Queen de Henry y Daniel Purcell (1695)

Of Birth, State, Wit, Strength, Courage, How'rd presumes,
And in his Breast wears many Montezumes
(Andrew Marvell, Last Instructions to a Painter, 1667)

Basada en la pieza teatral homónima de John Dryden y Robert Howard (1664), la versión semioperística ${ }^{10}$ de The Indian Queen escenifica la degradación y la posterior rehabilitación de un general de origen oscuro al servicio del Ynca ${ }^{11}$ llamado Montezuma. Tras numerosas intrigas amorosas, Montezuma logrará vencer a los tiranos mexicanos Traxalla y Zempoalla (enemigos del Ynca y enamorados respectivamente de Orazia, la hija de éste, y de Montezuma) y, tras descubrirse su ascendencia real, alcanzar la mano de la princesa incaica.

En su «Connexion of The Indian Emperour, to The Indian Queen», Dryden aludía a las fuentes cronísticas como fuente para The Indian Emperour ${ }^{12}$, lo que permite suponer, sin más, que también tuvo acceso a ellas para la redac-

10 Género musical del barroco inglés, las semióperas (semi-operas o dramatick operas en inglés) alternaban partes declamadas con partes de canto y de baile. La semiópera The Indian Queen introduce una serie de alteraciones (condensaciones, transposiciones, supresiones e inserciones) respecto del texto dramático que modifican el sentido de la pieza teatral. Para resumir, podría decirse que el libretista de la semiópera trastocó la relación entre la ilegítima reina india Zempoalla y Montezuma (que, al igual que el Ynca y Acacis, no cuenta con ninguna sección cantada) para conceder una mayor relevancia a la primera, suprimiendo, junto a las de Montezuma, algunas de las repetidas evaluaciones negativas acerca de ésta (véase Núñez Ronchi, 2003: 102-104), por lo que The Indian Queen acabó mereciendo su justo título. La rehabilitación relativa de Zempoalla frente a la figura de Traxalla se deba tal vez a que, en las crónicas, los tlascaltecas aparecen sobre-evaluados frente a los cempoaltecas. Así por ejemplo, como lo ha notado B. Keen (1990: 176) y lo recuerda Maehder (2008: 67), Solís atribuye a Tlascala poco menos que las virtudes de una república romana, con su consejo de estado, su senado y sus elocuentes tribunos (1684: 137-139, lib. II, cap. 16). Los libretos de las grabaciones de The Indian Queen incluyen sólo las partes cantadas, por lo que es difícil hacer sentido de ellos.

11 La elección del nombre de Montezuma para un héroe peruano (si bien en la obra se precisa que se trata de un «extranjero») puede parecer desconcertante, sin más. En Alzire de Voltaire (1736), Montèze también aparece como natural del Perú. Por haber sido tratadas en otros trabajos (Núñez Ronchi, 2003; 2012), dejaremos de lado todas las críticas que han abrumado injustamente a los libretistas, haciéndolos culpables de tergiversar la Historia: en efecto, el discurso historiográfico y el operístico, junto con sus respectivos géneros, no comparten las mismas constricciones productivas ni el mismo régimen interpretativo.

12 Dryden, 1956-2002b: 28. 
ción de The Indian Queen. En cualquier caso, los compendios de Richard Hakluyt (Principall Navigations, 1589 y Principal Navigations, 1598-1600) y de Samuel Purchas (Pilgrimages, 1613-1617 y Purchas his Pilgrimes, 1625 respectivamente) incluían extractos traducidos al inglés de varios autores ${ }^{13}$. A disposición de Dryden estaban, al menos teóricamente, otras versiones inglesas parciales ${ }^{14} \mathrm{o}$ íntegras ${ }^{15}$ de textos coloniales españoles. El polígrafo inglés pudo asimismo haber apelado a alguna traducción francesa contemporánea de Gómara ${ }^{16}$, por ejemplo, la de Martin Fumée (1584), basada a su vez en la traducción italiana de Agostino di Cravalix (1576), y a los Essais de Michel de Montaigne, en traducción de John Florio, uno de los libros predilectos de Dryden ${ }^{17}$, o haber tenido acceso a otros textos coloniales en traducción latina, italiana o incluso española, aunque parece poco probable que leyera con suficiente fluidez este último idioma ${ }^{18}$. El musicólogo alemán Jürgen Maedher proporciona un escueto pero interesante panorama general de la difusión de los distintos textos cronísticos en el ámbito europeo:

Those writers who had openly criticized the Spanish conquest-for example, Bartolomé de las Casas, Bernardino de Sahagún, and their successors [Motolinía, Alonso de Zorita, Jerónimo Román y Zamora, Diego Durán] did not enjoy a publishing success on Spanish or Spanish-dominated soil, nor in Italy generally, whereas these same works were often translated and distributed in Protestant countries $[\ldots]$. On the other hand, the accounts of the eye witnesses, Cortés's Cartas de relación and the Historia verdadera by Bernal Díaz del Castillo, continuously enjoyed the favor of the educated public $[\ldots]^{19}$. A third category of book, written by committed advocates of the Spanish cause and representing the official historiography as written against what were seen as derogatory publications im-

13 José de Acosta, Hernando de Alarcón, Nuño Beltrán de Guzmán, El Códice Mendocino, Francisco de Coronado, Hernán Cortés, Antonio de Espejo, Gonzalo Fernández de Oviedo, el Inca Garcilaso de la Vega, Antonio de Herrera, Sancho de la Hoz, Francisco de Jerez, Bartolomé de Las Casas, Francisco López de Gómara, Cristóbal de Mena, Marcos de Niza, Alvar Núñez Cabeza de Vaca, Francisco Ruiz, Hernando de Soto y Francisco de Ulloa.

14 Pedro Mártir, Bernal Díaz del Castillo, Hernán Cortés, Antonio de Herrera, Pedro Cieza de León, Gonzalo Fernández de Oviedo y Agustín de Zárate.

15 Cristóbal de Acuña, Francisco López de Gómara, Martín Fernández de Enciso, Bartolomé de Las Casas, Juan González de Mendoza.

16 MacMillan, 1950: 367-368 y 370.

17 Smith y MacMillan, 1966: 310-311.

18 A este respecto, véanse: Gaw, 1917: 16. Allen, 1935: 5. Hume, 1905: 295-96. Steele, 1975: 77.

19 Téngase en cuenta, sin embargo, como lo señala Zavala (1994: 141), que la obra de Bernal «se leyó principalmente en España y en la América española. La obra del historiador Antonio de Solís, Historia de la Conquista de México, publicada en 1684, se tradujo al italiano en 1699, al inglés en 1724 y al francés en 1759». 
pugning the honour of the Spanish crown, started to dominate the book market at the end of the seventeenth century [Fernández de Oviedo, Ginés de Sepúlveda, López de Gómara, Solís y Rivadeneyra] ${ }^{20}$.

Pero, aunque presumiblemente basada en los textos coloniales mencionados arriba, la pieza heroica y su contrapartida operística se distancian de ellos en varios aspectos. Por un lado, y ello se debe a consideraciones de orden discursivo y genérico, hay que insistir en la novedad temática que supuso The Indian Queen en las letras inglesas, pues la pieza asociaba por primera vez los temas del drama heroico, Amor y Honor, a actores americanos ${ }^{21}$. Por otra parte, y en esto se diferencia de sus sucesoras, la re-evaluación positiva del indio se hace también evidente en el privilegio absoluto de que goza el universo de interlocución, de asunción y de interacción indígena en la pieza y en la ópera ${ }^{22}$. Así, a la vez que presenta una constelación íntegramente indígena de personajes, desde el punto de vista del componente dialéctico ${ }^{23}$ y de los contenidos evaluativos, la pieza inglesa se distancia de ellas al conceder a Montezuma el rol de agente restaurador de la legitimidad política y religiosa ${ }^{24}$, y ello a pesar de que la pieza comparte con las crónicas en lengua española la misma repulsa por las prácticas sacrificiales ${ }^{25}$.

Por otra parte, para los asiduos lectores de las crónicas españolas, la novedad de la obra dramática The Indian Queen consiste en la peculiar resemantización de algunos de los gentilicios y nombres propios de la pieza; frente a las crónicas que no cesaban en afirmar el carácter determinante (pero siempre subordinado) de la ayuda brindada por los leales «amigos» cempoaltecas y tlascaltecas en el proceso de conquista, The Indian Queen invierte las evalua-

20 Maehder, 2008: 65-66. Nuestros agradecimientos al autor por su generosidad.

21 «An Heroick Play ought to be an imitation, in little of an Heroick Poem, and, consequently, Love and Valour ought to be the subject of it» (Dryden, 1956-2002c: 10).

22 Exceptuando una alusión en el Prólogo (vol. 14) y otra en el Epilogo (vol. 1 y vol. 10), que interpretamos como una referencia a los españoles y a los ingleses respectivamente.

23 El componente dialéctico de un texto da cuenta de la sucesión del tiempo representado, independientemente de su manifestación lineal. Los encadenamientos estereotipados de procesos son generalmente propios de un género (por ejemplo, en la tragedia).

24 «King Montezuma their loud shouts proclaim, / The City rings with their new Sovereings name, The Banish'd Queen declares he is her Son, / And to his succor all the people run» (Dryden, 1956-2002a: acto V, escena i, versos 192-95).

25 «ZEMPOALLA: That Prince upon whose ruines I must rise / Shall be the Gods, but more my sacrifice: / They with my slaves in Triumph shall be tyed, / While my devotion justifies my pride» (Dryden 1956-2002a: acto II, escena ii, versos 32-35) «THE CONJURER: You twice Ten Hundred Deities, / To whom we daily Sacrifice» (Dryden, 1956-2002a: acto III, escena ii, versos 64-65). 
ciones atribuidas a Zempoalla y Traxalla respecto de sus homónimos cronísticos, al tiempo que los singulariza ${ }^{26}$.

Suficientemente significativa nos parece a este respecto la escala evaluativa que presenta la «Segunda relación» de Hernán Cortés, que sitúa a los tlaxcaltecas por detrás de la Providencia, de los españoles y de los équidos, en ese orden: «En este desbarato [la «Noche Triste», como la denominó Gómara] se halló por copia que murieron ciento cincuenta españoles y cuarenta y seis yeguas y caballos y más de dos mill indios que servían a los españoles» (1993: 282 y 159-309). Asimismo, afirma Cortés más adelante, «no teníamos después de Dios otra seguridad sino la de los caballos» (1993: 285). En cuanto a «Zempoalla», como lo señala Palm, la reina mexicana «ostenta el nombre de la ciudad totonaca que fue la primera en acoger a Cortés» (1990: 91). Pero nada dicen las crónicas acerca de una reina cempoalteca, y concuerdan más bien en mencionar a un cacique entrado en carnes que los conquistadores apodaron «el gordo», con quien Cortés pronto se aliaría. La transexuación de este cacique responde sobre todo a las necesidades de las normas del género textual y a las oposiciones que recorren The Indian Queen, de suerte que en la pieza lo femenino aparece frecuentemente asociado a México, mientras que lo masculino tiende a conectarse al Perú. Ello se debe posiblemente al hecho de que «Mexique» -tal vez por influencia de «Nueva España»- era palabra femenina en el francés del siglo XVII (véase el Polexandre de Marin Le Roy de Gomberville). En cuanto a Traxalla, poco debieron de importarle a Dryden las informaciones consignadas por Gómara sobre el origen del nombre de los

${ }^{26}$ La cuestión de las denominaciones y de los predicados es primordial para la semántica intrepretativa, entre otras cosas porque permite establecer las evaluaciones y lo que se ha denominado las zonas antrópicas del texto -(Rastier, París, 2007). Por ejemplo, Bernand y Gruzinsky (1991, vol. 1: 439) han notado que, para la conquista del Perú, los textos de época no hablan nunca de «soldados», sino de «españoles», de «cristianos», lo que permite entroncar la Conquista con la Reconquista (Ballesteros 1984: 79n). En su Brevísima relación, Las Casas nombra a los españoles mediante un genérico «ellos» (1992: 36), del que se distancia, o mediante apelativos perifrásticos: «un infelice gobernador» (1992: 27), «el capitán de los españoles» (1992: 40), «aqueste capitán tirano» (1992: 44). Por el contrario, los caciques y otros dignatarios indígenas son designados mediante un antropónimo: Guarionex, Guacanagarí, Caonabó, Anacaona y Higuanamá en la Española (1992: 16-21), Hatuey en Cuba (1992: 24), «nuestro universal señor Motenzuma» en la Ciudad de México (1992: 40). Otro representante de la llamada «Leyenda Negra», el italiano Girolamo Benzoni, cuya Historia del Nuevo Mundo se publicó en 1565, ofrece por su parte la particularidad, muy moderna, de incluirse a sí mismo dentro de la clase semántica que denigra: «Viendo pues los indios nuestra frenética codicia y desmesurada avaricia, hay algunos que, cogiendo un trozo de oro en la mano dicen: 'Este es el dios de los cristianos'» (Benzoni, 1989: 230-231, lib. II). El énfasis es nuestro. 
más preciados aliados de Cortés, sobre las características geográficas del señorío o las costumbres de sus moradores. Importaba ante todo proceder a una resemantización de su nombre, pues según había afirmado su propio creador, «the Taxallan-Indians [were] the inveterate Enemies of Montezuma» ${ }^{27}$. Sin olvidar, por supuesto, el trágico fin que el texto reserva a estos dos traidores, pues Traxalla perecerá a manos de Montezuma y Zempoalla acabará dándose la muerte ${ }^{28}$.

En 1665, John Dryden publicó en solitario la continuación de The Indian Queen, que tituló The Indian Emperour, y que versa exclusivamente sobre la conquista de México. En ella, si bien Cortés vence a la nación mexicana y obtiene la mano de la princesa mexicana, prácticamente el resto del elenco de personajes indígenas perecerá a manos de los españoles, incluido Montezuma, mientras los dos únicos supervivientes mexicanos de la obra preferirán huir hacia las inhóspitas tierras del norte.

El «Dramma Per musica» Motezuma de Antonio Vivaldi (1733)

«No me joda con la Historia en materia de teatro» (Alejo Carpentier, Concierto barroco, 1974)

Si bien no tenemos constancia de ello, el tema de la Conquista de México y la solución de la unión entre los pueblos que se daba en The Indian Emperour (1665), continuación de The Indian Queen, debió de parecerles suficientemente atractiva al llamado "padre rojo» Antonio Vivaldi y a su libretista Alvise Giusti ${ }^{29}$, quien retomó el argumento para su «dramma per musica»

27 (Dryden, 1956-2002b: 27). Es posible asimismo que la variante drydeniana se diera por influencia de «Taxalla», príncipe italiano favorito del gran Tamerlán, cuyo nombre aparece en Histoire indienne d'Anaxandre et d'Orazie (1629) de Le Métel de Boisrobert, de donde Dryden había tomado prestado el nombre de Orazia.

28 Recuérdese que en Inglaterra no tenían vigencia las prohibiciones tridentinas en materia de suicidio o «desesperación».

29 «The poet of the opera is presumed to have been the young Luigi Giusti, a Venetian lawyer who later became an official of the Austrian government in Milan and associasted himself with the Enlightenment movement in Lombardy, thus coming into contact with the circle of Cesare Beccaria. This fact sheds an interesting light on the political dimension of the author's treatment of the historical subject of the fall of the Aztec empire and of the imperialistic politics of the Spanish Conquistadors» (Voss, 2008: 3). En cuanto al componente temático, Fabbri explica cómo los libretistas venecianos, entre ellos Aurelio Aureli, debían ingeniárselas para satisfacer a un público exigente deseoso de novedades temáticas: «So ch’il 
Motezuma, basándose, según afirma él mismo, en la Historia de la Conquista de México de Solís y Rivadeneyra, publicada en Madrid en 1684 y traducida al italiano desde 1699 .

En efecto, es preciso mencionar, durante todo el XVII, la importancia de Venecia como foco más importante de la ópera en Italia, si bien ya a comienzos del siglo XVIII el estilo llamado «napolitano» empezaba a rivalizar con la ópera veneciana. A la vez, es necesario destacar la importancia de Italia y de Venecia en particular como centros de impresión y de difusión de libros sobre América, de tal modo que, para finales del siglo XVI, Gómara, Cieza de León y Acosta ya habían sido traducidos íntegramente. Así, la segunda parte de la crónica de Gómara (1552) había sido traducida por Agustino de Cravaliz en 1556 (reed. 1560, 1556, 1560) y por Lucio Mauro en 1557. Junto con la traducción de Gómara, Ziletti (1555) había sacado de sus imprentas venecianas la traducción de la Crónica del Perú (1553) de Pedro Cieza de León (primera parte). Entre 1563 y 1606, Ramusio daba a las imprentas venecianas sus Navigationi et viaggi, cuyo tercer volumen correspondía a las gestas de Colón, Cortés, Pizarro y «oltri valorosi capitani», mientras Girolamo Benzoni publicaba en 1565 su exitoso Novae novi orbis historiae libri tres. Solís y Rivadeneyra, que se quiso continuador de Antonio de Herrera, escribió en parte su crónica para lavar el honor de los españoles: «los Estrangeros [Teodoro de Bry, Girolamo Benzoni, Jean de Laert, a los que habría que sumar a Las Casas], que no pueden sufrir la gloria de nuestra Nacion» ${ }^{30}$, presentaban «grande ossadia, y no menor malignidad, para inventar lo que quisieron contra nuestra Nación; gastando libros enteros» ${ }^{31}$. En cuanto al libreto de la ópera, Vickers señala que:

Le texte de Giusti est librement fondé sur la conquête du Mexique par Hernán Cortés (Fernando) et met l'accent sur la chute de l'empereur mexicain Montezuma (Motezuma). Giusti intégra certains détails historiques, encore que dans sa préface au livret il fasse de moqueuses allusions à la méfiance que lui inspirent les sources ${ }^{32}$.

gusto del popolo di Venezia à arrivato a tal segno che non sa più che bramar di vedere, né $\mathrm{i}$ compositori sanno piú che inventare per sodisfar al capriccio bizarro di quella cità» (Aurelio Aureli, en Perseo, 1665, citada en Fabri, 1990: 274).

30 Solís, 1684: s.p.

31 Ibidem: 2a, lib. I, cap. 1.

32 «[Solís] Ne scrisse con minor sospetto di tutti gl'autori la famosa penna del De Solis, e quantunque giudicato il più interessato nelle glorie di quest'eroe, nulladimeno io lo giudico il più sincero» (Giusti, 1733: 2). Con todo, la afirmación de Giusti no ha sido siempre interpretada como una crítica a su fuente histórica: «Nel breve Argomento que precede il lbretto, l'autore [Giusti] riferisce di avere utilizzato come fonte appunto l'Historia del Solís, ma lo ha 
Il s'autorisa de considérables licences artistiques en concevant des incidents qui servent à adapter le sujet au théâtre ${ }^{33}$.

En 1690, a pesar del fracaso en Roma de una ópera de Pietro Ottoboni sobre un tema similar ${ }^{34}$, el Motezuma de Vivaldi se estrenó en Venecia en el Teatro Sant'Angelo, el 14 de noviembre de 1733. A partir de ese momento, la música se da por desaparecida. Tanto es así que en 1992, para conmemorar el V centenario del Descubrimiento, Jean-Claude Malgoire realizó un pasticcio de la música sin referirse a la partitura, estrenado el 8 de mayo en Monte Carlo y con Ariel García Valdés como director de escena: «Malgoire accorded scrupulous respect to the libretto of 1733 in respect of the casting and the unfolding of the plot, with the sole exception of an exchange of vocal registers between Motezuma and Fernando in relation to the original cast ${ }^{35}$. En 2002 se redescubre la partitura, incompleta, en un archivo berlinés secuestrado por el ejército ruso durante la Segunda Guerra Mundial. Finalmente, la ópera ha podido ser restituida casi en su integralidad en la grabación de 2006 (Deutsche Grammophon, dir. Alan Curtis), completando Alessandro Ciccolini los recitativos faltantes ${ }^{36}$.

Tras numerosas peripecias, al final del drama operístico de Vivaldi, y al igual que ocurre en el último acto de The Indian Emperour, continuación de The Indian Queen, la princesa mexicana Teutile - «ipero si Teutile, carajo, era un general mexicano!», se exclama reiteradamente el indiano en Concierto Barroco de Alejo Carpentier ${ }^{37}$ - celebra sus esponsales con Ramiro, hermano de Hernando, bajo los auspicios paternos. De modo que en la ópera de Vivaldi no sólo no muere Montezuma, sino que además bendice la unión de los amantes $\mathrm{y}$, de alguna manera, la unión entre los dos pueblos ${ }^{38}$. Como lo indi-

fatto in modo decisamente più disinvolto di quanto lascerebbe credere la sua timida ammissione di essere stato a qualche cambiamento per esigenze della scena» (Fantoni, 2000: 179-80).

33 Vickers, 2006: 49.

34 Il Colombo ovvero l'Idia Scoperta (Roma, Teatro di Tordinona, 28-12-1690). En sus Memorias, Philippe-Emmanuel Coulanges, que se encontraba por aquel entonces en Roma, no duda en denigrar la ópera, celebrando que las noticias de la peste pusiera fin a la temporada operística: «Nous avons eu ce carnaval / Une pièce très execrable, / Faite par Ottobon, poëte détestable ... / Quel opéra maudit, où l'on crioit merveilles! / Que Christophe Colomb m'a lassé les oreilles! / [...] / Le grand bruit de la peste, en tous lieux répandu, / A fait cesser cette musique» (Coulanges, 1820: 284-85).

35 Delaméa, 2008: 176.

36 Vickers, 2006: 57.

37 Carpentier, 2011: 214.

38 Teutile «è il personaggio a cui il destino riserva la sorte più difficile e complessa, quella di essere partecipe di due mondi, di due culture, di due affetti ugualmente importanti» (Fantoni, 2000: 175-180. 
ca Maehder ${ }^{39}$, este final feliz, en contradicción evidente con las fuentes históricas ${ }^{40}$, ha suscitado numerosos comentarios por parte de los críticos, que suelen resolver la aparente paradoja apelando a las normas del género operístico ${ }^{41}$ : el lieto fine, o final feliz, era de casi obligado cumplimiento en la opera seria, al menos hasta finales del siglo XVIII ${ }^{42}$.

Ante la prescripción genérica del lieto fine, las críticas a la conquista y a la codicia española se manifiestan en la ópera por otros medios, menos evidentes, a la vez que se resalta el carácter trágico de los personajes indígenas. Así, aunque escenifique la unión sentimental entre los dos pueblos, la pieza no cuenta con ninguna escena tradicional de amor ${ }^{43}$; al mismo tiempo, dado que Motezuma es el último personaje en verse adjudicado un aria, este aparece como el protagonista trágico de la ópera ${ }^{44}$. A la vez, su esposa Mitrena no duda en descalificar al conquistador Ferdinando ${ }^{45}$ que, según algunos críticos ${ }^{46}$, actúa impelido por motivaciones dudosas, pero ostentando sin embargo al mismo tiempo el rol de primo uomo en la ópera ${ }^{47}$.

39 Maehder, 1993: 158.

40 Las fuentes españolas (Cortés, Bernal Díaz, Gómara, Aguilar, Solís), aunque varían sobre otros detalles, suelen acordarse sobre la muerte por lapidación de sus súbditos, a diferencia de lo que indican las fuentes indígenas o indigenistas (Sahagún, Durán, Las Casas, Acosta, Códice Ramírez, Códice Florentino, Códice Moctezuma, Ixtilxóchitl, Chimalpaín), que atribuyen su muerte a los españoles.

41 Maehder, 2008: 72.

42 Maedher, 1993: 160. La muerte de Montezuma en la primera versión de la ópera de Vittorio Amadeo Cigna-Santi (Turín, 1765) no debe considerarse como una instancia de fin trágico, ya que el protagonista de esta pieza es Cortés, o incluso la religión católica. Por lo que se refiere a la versión de esta misma ópera musicalizada por Baldassare Galuppi (Venecia, 1772), Maehder añade que «On Venetian territory, though, there was no need for a glorification of monarchy and even less interest for a pro-Hispanic final chorus. The extremely short Act III of Galuppi's score deals with the underlying political conflict in a very generic way, concentrating instead on the love relationship between Erismena and Motezuma». Maehder, 1993: 146-184.

43 «[...] dans la lutte pour le pouvoir, ils ne trouvent pas le temps de s'aimer-peut-être une métaphore pour le fossé infrachissable entre les deux continents» (Vickers, 2006: 54).

44 Voss, 2008: 9.

45 Maehder, 2008: 70.

46 Curtis, entrevistado por Vickers, 2006: 54.

47 Así por ejemplo, en la primera representación del 14-11-1733, Ferdinando fue interpretado por el joven Francesco Bilanzoni, soprano castrato, mientras el barítono Massimiliano Miller interpretaba a Motezuma (Voss, 2008: 5-6). En el pasticcio de J.-Cl. Malgoire, en cambio, el rol de Motezuma fue atribuido al contratenor Dominique Visse (Astrée, 1992), y al barítono Vito Priante en la grabación de Curtis (Deutsche Grammophon, 2006). 
Así pues, tal vez sean precisamente estas contradicciones irresueltas las que, al igual que ocurre con The Indian Emperour, permitan renovar casi incesantemente las interpretaciones de la obra ${ }^{48}$.

\title{
Les Indes Galantes, Ballet héroique ${ }^{49}$ De Jean-Philippe Rameau (1735)
}

«Il me semble qu'il faut être aveugle pour ne pas voir que la propriété des biens (je ne dis pas celle des femmes) est la seule source de tous les désordres qui troublent la Société des Européens» (Baron de Lahontan, Dialogues, 1704)

\author{
En Francia, el tema indiano amoroso ${ }^{50}$ retorna a los escenarios de la mano \\ de Jean-Philippe Rameau con un libreto compuesto por Louis Fuzelier. En-
}

48 «Le but de Giusti n'est manifestement pas de dépeindre le traditionnel triomphe du christianisme sur les barbares. Lorsque Mitrena proteste contre les atrocités de Fernando, celui-ci se défend en disant que ses massacres sont 'vergini', honnêtes et purs, et que ses armes sont utilisées uniquement pour défendre le 'droit du ciel et de la terre'. Cette référence indirecte est la seule allusion à son christianisme supposé, et ne le place certainement pas sous un un jour très favorable. Il ne donne pas à la 'Conquête' des excuses religieuses ou même politiques, et ne tente pas de masquer la violence, la brutalité et l'avidité qui accompagnent inévitablement de telles entreprises» (Vickers, 2006: 54). «This libretto has exercised fascination on account of its unusual geographical setting and original subject, and especially for its bold words that denounce the violence of the earliest European colonization with a force that for its time appears iconoclastic» (Délaméa, 2008: 169). "Although the Spanish leader Fernando (the historical Hernán Cortés) is treated as the glorious hero of the drama, his character is not free form arrogance and cruelty, as is observed by Motezuma's wife Mitrena during her diplomatic conversation with him» (Voss, 2008: 3).

49 «De temps en temps des tentatives sont entreprises pour rendre l'intrigue plus dramatique. Elles sont indiquées par l'épithète d'héroïque ajoutée au titre et c'est à cette catégorie de ballet héroïque qu'appartient Les Indes Galantes» (Girdlestone, 1962: 332)

50 El tema americano había sido tratado ya por Du Rocher en su pieza teatral L'Indienne amoureuse (París: Corrozet, 1635), por Alexis Piron en Fernand Cortez (1744), por Louis Ferrier de la Martinière en Montezume, hoy perdido (1702), e incluso abordado musicalmente en la Découverte du nouveau monde de Jean-Jacques Rousseau, ópera compuesta en Lyon en 1741, basada presumiblemente en su tragedia homónima y cuyo libreto fue aparentemente escrito por el propio Rousseau. Pero como lo señala Ballón Aguirre (2001: 39) «Las Indias Galantes es la primera ópera de factura gala en la que las aventuras de los 'dioses míticos' sobre las que anteriormente se fundamentaba todo argumento, son arrinconadas en un lugar muy modesto (el prólogo) y dejan el motivo central de las intrigas (en las cuatro entradas) a sucesos acaecidos a 'héroes históricos'», si bien es cierto que la ópera-ballet, a diferencia de la ópera trágica, no recurría necesariamente a los héroes mitológicos (Girdlestone, 1962: 331). 
mendado en numerosas ocasiones, el texto definitivo comprende cuatro entradas, de las cuales la segunda y la última trascurren en el Nuevo Mundo. Entre sus fuentes, Rameau ${ }^{51}$ menciona a Garcilaso de la Vega, traducido al francés por J. Baudoin (París: Augustin Courbe, 1633; reed. 1650; Amsterdam, 1704), a Solís, sacado en las prensas parisinas de Villery en 1691, y a Zárate, traducido al francés por Samuel de Broë, Seigneur de Citry et de la Guette en Amsterdam, en 170052. Ballón Aguirre (2001: 123) menciona dos reimpresiones más (París: Osmot, 1706 y 1716), y pueden localizarse varias ediciones y reimpresiones hasta 1719. Para 1735, los textos de Gómara (trad. 1568), de Benzoni (trad. 1600), Acosta (trad. 1606) y Herrera (trad. 1622) contaban ya con traducciones íntegras al francés. Para la cuarta entrada, la fuente más probable es la del Baron de Lahontan, Dialogues de M. le Baron de Lahontan et d'un Sauvage dans l'Amérique (Amsterdam: Chez la veuve de Boteman et se vend à Londres chez David Mortier, 1704). En cuanto al argumento de la segunda entrada, titulada «Les Incas du Pérou», he aquí lo que resume el crítico peruano Ballón Aguirre:

Don Carlos, oficial español, y Phani, princesa peruana (palla), se aman. Huáscar, gran sacerdote del Sol, ama también a Phani y trata de vengarse de manera pintoresca, en todo caso poco francesa ${ }^{53}$ ( sic), provocando la erupción de un volcán (escena quinta) durante la fiesta del Sol (Inti Raimi) [...] Carlos logra socorrer a Phani mientras que Huáscar es sepultado por las rocas ${ }^{54}$.

El crítico peruano asegura que, con el objetivo de obtener los favores de la princesa Phani, las actuaciones tanto de Huascar como de Carlos se declinan ambas bajo el signo de la impostura, al pretender engañar el primero a Phani con un «bárbaro artificio» (la erupción del volcán en la entrada IV), y Carlos, porque deja en la ignorancia ${ }^{55}$ a Phani respecto de la superioridad técnica y militar de los españoles (que Phani considera como «milagros») ${ }^{56}$. Sin embargo, esta caracterización de Carlos suscita ciertas reservas, dado que

\footnotetext{
51 Rameau, 1736: vi.

52 Pueden consultarse, en la crónica de Zárate, el lib. I, cap. IX y el lib. II, caps. X y XI, sobre las contiendas entre Atahualpa y Huáscar.

53 Como lo señala Ballón Aguirre, el topos proviene de Chinard: «[...] de façon pittoresque, et en tout cas peu française» (Chinard, 2000: 233).

54 Ballón Aguirre, 2001: 28.

55 «En cambio, los 'milagros' de los conquistadores quedan sin explicación para Phani y Huáscar, Carlos aprovechará esta ignorancia para enarbolarlos a manera de argucia semi-simbólica dirigida a lograr su embuste» (Ballón Aguirre, 2001: 49).

56 «PHANI: Téméraire! Que dites vous ! / Révérez leur puissance, et craignez leur courroux. Pour obtenir vos hommages, / Faut-il des miracles nouveaux? / Vous avez vu, de nos
} 
su supuesta impostura no es nunca afirmada por el propio Carlos, ni desenmascarada por Phani, ni presentada como tal por el enunciador (por ejemplo en las didascalias) que, al contrario, sí reprueba los actos del sacerdote Huas$\operatorname{car}^{57}$, refrendando así la condena que de sí mismo hace el propio Huascar ${ }^{58}$. El reparto vocal original (tesitura de tenor para Carlos y registro de bajo para Huascar $)^{59}$ parece confirmar nuestra interpretación y otorgar el rol de villano a Huascar.

Tal vez podría buscarse entonces la dimensión crítica de esta obra en los enunciados devaluativos proferidos por Huascar acerca de los españoles: «C'est l'or qu'avec empressement, / Sans jamais s'assouvir, ces barbares [los españoles] dévorent. / L'or qui de nos autels ne fait que l'ornement / Est le seul Dieu que nos tyrans adorent ${ }^{60}$. Así, «l'imposture cachée sous le manteau sacré de la Religion» que el enunciador reprocha a Huascar61 sea tal vez la misma que puede atribuirse a los españoles y más exactamente a $\operatorname{Carlos}^{62}$, cuyo nombre recuerda extrañamente al del Emperador bajo cuyo reinado se conquistó el Perú. Sin embargo, el hecho de que estos enunciados no sean

rivages, / Leurs villes voler sur les eaux, / Vous avez vu, dans l'horreur de la guerre, / Leur invincible bras disposer du tonnerre...» (Rameau, 1736: acto II escena iii).

57 Dice el enunciador en la introducción al libreto, a propósito de Huáscar: «Un Sacrificateur payen, aveuglé par la jalousie \& guidé par la fureur, se sert de ce dangereux Phénomène pour réussir dans ses projets criminels, Quels artifices ne risque pas l'Amour entraîné par le desespoir, \& l'imposture cachée sous le manteau sacré de la Religion ?» (Rameau, 1736: $\mathrm{v}$-vi).

58 «HUASCAR: Abîmes embrasés, j'ai trahi les autels» (acto II, escena viii). El caso de la elección de Huáscar, generalmente presentado por los cronistas como el legítimo sucesor de Huayna Cápac (Gómara, Historia general de las Indias, 1979: 175-176, cap. CXVI), aparece tal vez como similar al de «Traxalla» y «Zempoalla», conforme al topos de que «los enemigos de mis enemigos son mis amigos», puesto que Huascar contendía con su medio hermano Atahualpa por la supremacía del imperio. Algo similar comenta Carlos Araníbar (Araníbar, 1995, vol 2: 764) con respecto al agustino Román y Zamora que, por defender a Atahuallpa de los Pizarro, termina convirtiendo a Huáscar en el «malo de la historia».

59 Interpretados respectivamente por el contratenor Pierre Jélyotte y el bajo Claude Chassé de Chinais (Kaminsky, 2011: 194). Girdlestone afirma asimismo que Huascar es «le scélérat de l'histoire» (Girdlestone, 1962: 341).

60 Rameau, 1736: acto II, escena iii.

61 Ibidem: vi.

62 Ballón Aguirre (2001: 130) indica que «El nombre español 'Carlos’ por el epónimo Carolus Magnus (Carlo Magno) significa 'conquistador', pero también 'hombre corriente del pueblo'». Sin embargo, su etimología más común es «hombre libre» o incluso «viril», si

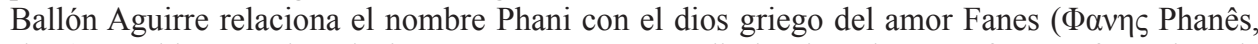
'luz'), también puede relacionarse con «Fanny», diminutivo de «Estefanía», femenino de «Esteban» que, a su vez, proviene del griego «Stéphanos», con el significado de «guirnalda», «corona», literalmente «lo que rodea, envuelve, ciñe». 
nunca desmentidos, pero tampoco confirmados, vuelve su valor de verdad indecidible.

La cuarta y última entrada, titulada «Les Sauvages», cuya acción tiene lugar en «un bosquet d'une forêt d'Amérique, voisine des colonies françaises et espagnoles» (IV), y en la que dos militares extranjeros, el celoso español don Alvar y el inconstante francés Damon se disputan los favores de la bella Zima, hija del jefe de la nación salvaje, quien sin embargo prefirirá entregar su corazón a su compatriota Adario ${ }^{63}$, es una adición tardía que aseguró el éxito definitivo de la ópera el 10 de marzo 1736, «lo que le valió los honores de la parodia ${ }^{64}$.

Coincidimos con Girdlestone y con Ballón Aguirre en que, en esta entrada, el esquema de valores ideológicos es sumamente esquemático y estereotipado $^{65}$, lo que, sin embargo, no le resta necesariamente interés, pues permite vislumbrar los topoi o axiomas sociales normalizados del texto, cuando no del género textual ${ }^{66}$. De cualquier modo, la repentina desaparición de los españoles en la sexta y última escena de esta entrada, en la que «Zima, Adario, Françaises en habits d'amazones, guerriers français et sauvages, sauvagesses et bergers de la colonie» se reúnen en el bosque antes de que los Salvajes ejecuten la Danza de la Gran Pipa de la Paz (acto IV, escena vi), deja pocas dudas sobre la suerte que esta entrada reserva a los españoles en las colonias americanas ${ }^{67}$.

63 «ZIMA: Je ne veux d'un époux ni jaloux ni volage. / (A l'espagnol) Vous aimez trop, / (au français) Et vous, vous n'aimez pas assez». (Rameau, 1736: acto IV, escena iii). «ZIMA: [...] Je vous [Adario] dois la préférence. / De vous à vos rivaux je vois la différence: / L'un s'abandonne à la fureur, / Et l'autre prend mon coeur avec indifférence. / Nous ignorons ce calme et cette violence. / Sur nos bords l'amour vole et prévient nos désirs» (Rameau, 1736: acto IV, escena v).

64 Zavala, 1994: 62. Les Indes Galantes se presentó trescientas veces entre 1735 y 1773 (Fürst, 1993: 22).

65 Girdlestone, 1962: 351, Ballón Aguirre, 2001: 50.

66 Así, podría verse en el celoso y asesino don José en Carmen de Bizet (libreto de Ludovic Halévy y Henri Meilhac, Opéra Comique, 3-3-1875), militar español al igual que don Alvar, una prolongación de este último. Damon - del griego $\delta \alpha \mu \omega v$, demonio tentador (Ballón Aguirre, 2001: 133n)-, es un personaje más hablador (Girdlestone, 1962: 352), extrovertido y fatuo (Girdlestone, 1962: 351) que Don Alvar.

67 «Au ballet prennent part Zima et Adario (les prétendants éconduits ayant disparu), et Françaises en habits d'Amazones, guerriers français et sauvages, sauvagesses, bergers de la colonie» (Girdlestone, 1962: 354-355). El énfasis es nuestro. 
Montezuma, «tragedia Per musica» de Carl Heinrich Graun (1755)

«Y todo esto pasó con nosotros.

Nosotros lo vimos, nosotros lo admiramos.

Con esta lamentosa y triste suerte nos vimos angustiados».

(Miguel León Portilla, Visión de los vencidos, 1959)

La última ópera que abordamos aquí es sin duda la más ideológicamente marcada ${ }^{68}$. Desestimando las prescripciónes relativas al lieto fine, Federico II el Grande, que escribió el libreto de Montezuma (Berlín, 6-01-1755) en francés que fue posteriormente traducido al italiano por Giampietro Tagliazucchi, no dudó en representar los grillos, la prisión y la ejecución (eso sí, ésta última fuera de escena, por cuestiones de decoro) del emperador azteca, y poner fin a la vida de su prometida Eupafórice y de los dos generales mexicanos, Pilpatoè y Tezeuco ${ }^{69}$. Montezuma había perecido ya bajo la pluma de Dryden en The Indian Emperour. Sin embargo, en esta ópera, Montezuma no fallecerá por mano propia como en el drama inglés ${ }^{70}$, sino por la mano culpable de Cortes, que nos es repetidamente presentado como un impostor criminal que logra, al menos hasta conseguir el objetivo de introducirse con sus hombres en el palacio imperial, engañar al pueblo y al emperador mexicano, que lo han acogido con los brazos abiertos ${ }^{71}$. Frente a todo un elenco de voces agudas $^{72}$, la tessitura de mezzo-soprano atribuida a Montezuma en la grabación de 1992 otorga al emperador mexicano toda su dimensión trágica.

68 Hay que señalar que La Brevísima relación de Bartolomé de las Casas conoció nada menos que cuarenta y dos ediciones europeas entre los siglos XVI y XVII (Fajardo, 1992: 6). Según Marion Fürst (1993: 23), la ópera de Graun está basada en la traducción francesa de Solís y Rivadeneira. Fernández Salek (1999: 30) ha apuntado que «Gracias a la imprenta, Alemania contribuyó en gran medida a la distribución de información de diferente género de relatos, existiendo básicamente cinco: hojas volantes y gacetas, descripciones de viajes por testigos visuales, complicaciones de viajes, cosmografías y crónicas y literatura ficticia».

69 Graun, 1904: 213, acto III, escena v.

70 Dryden, 1667: 64-65, acto V, escena ii.

71 Dryden, 1667: 78-83, acto II, escena ii.

72 Aunque la grabación, incompleta, a cargo de Johannes Goritzki (Capriccio, 1992), cuenta con un elenco de un alto, cinco sopranos y una mezzo-soprano, parece que «el papel de Moctezuma estuvo originalmente diseñado para Giovanni Tedeschi, uno de los mayores contraltos castrati de mediados del siglo XVII» -Jouve-Martín (Liverpool, 2010: 208). 
Así, mientras por un lado, fruto de su bondad ${ }^{73}$, el clemente y generoso Montezuma, su oficial del ejército imperial Tezeuco y el Coro son engaña$\operatorname{dos}^{74}$, por otro lado Pilpatoè, general del emperador, y Eupaforice, la reina tlascalteca prometida de Montezuma ${ }^{75}$, presienten en vano el peligro, creando en la ópera una topografía variable de acercamientos y distanciamentos tanto en el ámbito físico como sentimental (desencuentro entre Montezuma y su amada Eupaforice, que no logran nunca compaginar su ritmo amoroso ${ }^{76}$; introducción de las huestes españolas en el palacio imperial y posicionamiento frontal de los dos ejércitos ${ }^{77}$, cuyo correlato dramático-musical se manifiestan en la asimétrica constelación de personajes (siete en lugar de los seis u ocho

73 «Montezuma, che ne era lo Imperatore, gliene permise sotto la buona fede l'ingresso» (Graun, 1904: s. p.).

74 «NARVÈS. L'Imperator s'appresa, or che n'imponi? / CORTES: Di simular, di fingere, di dargli di rispetto e umiltà vani tributi. Ci conviene su l'orlo addor mentarlo del precipizio, in cui desio d'urtarlo» (Graun, 1904: 77, acto II, escena i). «MONTEZUMA: No, che un nume non sei, come la fama persuader mi volea» (Graun, 1904: 117, acto II, escena vii), «TEZEUCO: Sì, del soggiorno imperial / l'ingresso questo è, Signor ... Ma che pretendi ? Oh Dei...» (Graun, 1904: 101, acto II, escena v). Compárense estas dos intervenciones, inicial y final, del Coro: «CORO: Venite intrepide stranieri eroi, / tolti al terribile furor dell'onde. / Cari e pregevoli sarete / a noi e al nostro amabile Imperator» (Graun, 1904: 79-80, acto II, escena ii) y «CORO: Oh Cielo! ahí giorno orribile di delitti esecrabili. / O terra, che li tolleri, / apri le tue voragini. / Fuggiam, fuggiam dai barbari, / voi, giusti Dei, salvateci, / movetevi a pietà». (Graun 1904: 219-221, acto III, escena v), habiendo sido esta última intervención suprimida en la puesta en escena de Claudio Kuri Valdés en 2010.

75 «PILPATOE: [...] Ma frattanto, Signore, al lor coraggio, al lor fiero ardimento, che nin via sia riparo alcun pavento» (Graun, 1904: 23-24, acto I, escena ii), «EUPAFORICE: [...] Suona purtroppo il grido, che si vada appressando a questo impero / quello stuolo straniero / conquistador de' popoli vicini. [...] Se tremar mi vedi, non è per me, ch'io tremo: tremo per l'idol mio, per Moctezuma» (Graun, 1904: 35, acto I, escena iv).

76 «MONTEZUMA: [...] Amico, sia tua cura di prepare la solenne pompa del mio dolce Imeneo» (Graun, 1904: 27, acto I, escena ii), «ERISSENA: Regina, a che sì mesta? A che sospiri? qual amara cagion su gli occhi tuoi può quel pinato condur?» (Graun, 1904: 33, acto I, escena iv), «MONTEZUMA. [...] Ma tu, sposa fedele! ah, Eupaforice, il momento felice, che stringerci doveva d'indissolubil nodo, è quello... forse che dee per sempre separarci» (Graun, 1904: 166-167, acto II, escena i).

77 «CORTES: [...] oso pregarti che innalzi a tanto onore anche i compagni delle fatiche e de' perigli miei. Essi il loro destin m'ànno affidato. Senza taccia d'ingrato non posso abbandonarli. Alla tua reggia che mi posam seguir, Signor, permetti, e che godan con me de' tuoi diletti» (Graun, 1904: 85-86, acto II, escena ii), «Le guardie messicane saranno in buon ordine da una parte, Cortes dall'altra mette i suoi Spagnuoli in ordine di battaglia» (Graun, 1904, 101, acto II, escena v, Indicación escénica). 
habituales) y en la discrepancia entre la música alegre y festiva de la escena final $^{78}$ y las lamentaciones del coro ${ }^{79}$.

Como hemos visto, si bien es cierto que los españoles tienen un papel variable en estas óperas (desde su casi inexistencia en The Indian Queen hasta su absoluta condena en la ópera de Graun, pasando por una posición intermedia en las óperas de Vivaldi y Rameau), en cambio los indígenas, y más aún aquellos que las crónicas denigraban, aparecen positivamente evaluados en estas óperas, tanto en el ámbito sentimental como en el religioso y en el político.

Esto distancia estas piezas del género operístico en lengua española, prácticamente ajeno al temario americano hasta el siglo XIX ${ }^{80}$, y de la comedia áurea española, donde los personajes indígenas suelen aparecer devaluados, $\mathrm{y}$ donde raras, cuando no inexistentes, son las voces discordantes capaces de otorgar una dimensión crítica a la Conquista de América ${ }^{81}$. De hecho, los ataques y contrariedades que ha suscitado la reciente reposición de la ópera de Graun entre el público madrileño durante la conmemoración del Bicentenario de la Independencia de México en 2010 parecen corroborar la mencionada vacuidad de esas voces discordantes; tal vez, como lo afirma su director de escena Claudio Valdés Kuri, porque nuestro pasado colonial continúa abriendo heridas tanto en los pueblos conquistados como entre los conquistadores $^{82}$.

78 Maehder, 1992: 157.

79 Como lo indica acertadamente Fernández Salek (1999: 55): «Los personajes representados en la obra de Federico II el Grande son personajes colectivos, no presentan el problema de una sola persona, sino de toda una nación».

80 Lo que tal vez pueda considerarse como la primera ópera española de temática indiana (Las naves de Cortés de Ruperto Chapí, con libreto de Antonio Arnao) no se estrenó hasta 1874. Ciertamente, Ramón Carnicer había presentado Cristoforo Colombo en Barcelona en 1829, pero sobre un texto italiano de Felice Romani (1829). Dos años antes de Las naves de Cortés, en 1872, el propio Chapí estrenaba Vasco Núñez de Balboa, con libreto de Marcos Zapata, catalogada ésta como zarzuela.

81 Núñez Ronchi, 2013: 389-414.

82 «Si hay reacción es que no está resuelto. Hay un sentimiento de culpa que no tendría que existir, pero existe. La llaga está abierta. Yo sé que en México no está resuelto, pero entiendo que esté abierto mientras sigamos viviendo en una estructura tan jerárquica y con tantas diferencias. La condición del indígena frente al poder sigue siendo tan jerarquizada como lo fue en tiempos de la conquista. No creo que tampoco era el mundo perfecto que 
Ello permite a su vez relativizar nociones como la de «Leyenda Negra», entendida no como letanía de falsas y mentirosas infamias, sino como punto de confluencia de valorizaciones positivas o negativas, o incluso neutras. En efecto, dado que tanto las crónicas de Indias como estas óperas contienen numerosos rasgos evaluativos, acaba resultando arriesgado establecer una línea divisoria clara entre estos textos historiográficos y los textos legendarios, al menos en este aspecto. De tal forma que podría afirmarse que tanto la historia como las leyendas, sean negras o áureas, son finalmente interpretaciones de hechos que también son el producto de intepretaciones ${ }^{83}$.

\section{OBRAS CITADAS}

Acosta, José de, Historia natural y moral de las Indias, 1590, ed. Edmundo O'Gorman, México, D.F., Fondo de Cultura Económica, 2006.

Allen, Ned B., The Sources of John Dryden's Comedies, Ann Arbor, University of Michigan Press, 1935.

Araníbar, Carlos (ed.), "Índice analítico y glosario", Los comentarios reales, vol. 2, del Inca Garcilaso de la Vega, México, D.F., Fondo de Cultura Económica, 1995: 649-879.

Asch, David van, "Presentación", Henry y Daniel Purcell, The Indian Queen, CD, Naxos discográfica, 1995: 2.

Ballesteros, Manuel (ed.), La crónica del Perú, de Pedro de Cieza de León, Madrid, Historia 16, 1984.

Ballón Aguirre, Enrique, Desconcierto barroco, México, D.F., Universidad Nacional Autónoma de México, 2001.

Benzoni, Girolamo, Historia del Nuevo Mundo, Manuel Carrera Díaz (ed.), Madrid, Alianza, 1989.

Chinard, Gilbert, L'Amérique et le rêve exotique dans la littérature française au XVIIe et au XVIIIe siècle, Ginebra, Slatkine, 2000.

Cortés, Hernán, “Segunda relación”, Ángel Delgado Gómez (ed.), Cartas de relación, Madrid, Castalia, 1993: 159-309.

pintó Diego Rivera en los murales» («Montezuma y la Conquista, una historia no resuelta»). Sobre las reacciones suscitadas por la puesta escena de esta ópera a cargo de Valdés Kuri, véase asimismo la reseña en La Jornada, fechada el 18 de sept. de 2010.

83 «[...] les données sont bien ce qu'on se donne», «elles sont ainsi les résultats initiaux d'un processus d'élaboration -et leur traitement produit des résultats ultérieurs, dans un cycle susceptible de récursivité» (Rastier, 2010: 8-9). 
Coulanges, Philippe Emmanuel, Mémoires de M. de Coulanges, París, J. J. Blaise, 1820 .

Delaméa, Frédéric, "Vivaldi in scena: Thoughts on the Revival of Vivaldi's Opera", Vivaldi, 'Motezuma' and the Opera Seria: Essays on a Newly Discovered Work and its Background, Michael Talbot (ed.), Brepols, Turnhout, 2008: 169-202.

Dryden, John, “The Indian Queen”, H. T. Swedenberg et al. (eds. grales), The Works of John Dryden, vol. 8, Berkeley, University of California Press, 1956-2002a: 181-231.

Dryden, John, "Connexion of the Indian Emperour, to the Indian Queen", H. T. Swedenberg et al. (eds. grales), The Works of John Dryden, vol. 9, Berkeley, University of California Press, 1956-2002: 27-28.

Dryden, John, “Of Heroique Plays", H. T. Swedenberg et al. (eds. grales), The Works of John Dryden, vol. 11, Berkeley, University of California Press, 1956-2002: 8-18.

Dryden, John, The Indian Emperour, Londres, Printed by J. M. for H. Herringman, 1667.

Duviols, Pierre, Le miroir du Nouveau Monde: Images primitives de l'Amérique, París, Presses París Sorbonne, 2006.

Fabbri, Paolo, Il secolo cantante. Per una storia del libretto d'opera nel Seicento, Bolonia, II Mulino, 1990.

Fantoni, Giuliana, "Montezuma in alcuni libretti d'opera", Aldo Albonico y Fulvio Tessitore (eds.), Cultura latinoamericana, Istituto di Studi Latinoamericani, Pagani y Salerno, Edizioni del Paguro, 2000: 175-198.

Fernández Salek, Fabiola, "La crónica de la Conquista de México en una ópera barroca alemana: 'Montezuma' de Federico el Grande", Tesis de Doctorado de Arizona State University, 1999.

Fürst, Marion, "Mit Federschmuck und Friedenspfeife. Der Amerikaner in der Europäischen Oper des 17. und 18. Iahrhunderts", Musica, 47 (Kassel, 1993): 21-24.

Gaw, Allison, "Tuke's Adventure of Five Hours in Relation to the 'Spanish Plot' and to John Dryden", Allison Gaw (ed.), Studies in English Drama. First Series, Nueva York, D. Appleton, 1917: 17-35.

Girdlestone, Cuthbert, Jean-Philippe Rameau: sa vie, son oeuvre, París, Desclée de Brouwer, 1962.

Giusti, Alvise, Motezuma, Venecia, Marino Rossetti, 1733.

Graun, Carl Heinrich, Montezuma, Leipzig, Breitkipf \& Härtel, 1904.

Gildon, Charles, The Lives and Characters of the English Dramatic Poets, Londres, Printed for Tho[mas] Leigh and William Turner, 1699.

Hume, Martin, Spanish Influence on English Literature, Londres, Eveleigh Nash, 1905. 
Jouve-Martín, José Ramón, “Literatura, música e historia”, Bulletin of Hispanic Studies, 87/2 (Liverpool, 2010): 203-220.

Kaminski, Piotr, Lully, Rameau et l'opéra baroque français, París, Librairie générale française, 2010.

Las Casas, Bartolomé de, Brevísima relación, José Manuel Fajardo (ed.), Madrid, Cambio 16, 1992.

MacMillan, Dougald, "The Sources of Dryden's The Indian Emperour", Huntington Library Quarterly, 13 (Chicago, 1950): 355-370.

Maehder, Jürgen. "Mentalitätskonflikt und Fürstenpflicht: Die Begegnung zwischen Conquistador und mittelamerikanischern Hersscher auf der barocken Opernbühne", Micheal Walter (ed.), Text und Musik. Neue Perspektiven der Theorie, Munich, Fink, 1992: 131-179.

Maehder, Jürgen, “Cristóbal Colón, Motecuzoma II, Xocoyotzin and Hernán Cortés on the Opera Stage: A Study in Comparative Libretto History", Revista de Musicología, 16 (Madrid, 1993): 146-184.

Maehder, Jürgen, “Alvise Giusti's Libretto Motezuma and the Conquest of Mexico in Eighteen-Century Italian Opera Seria", Michael Talbot (ed.), Vivaldi, "Motezuma" and the Opera Seria: Essays on a Newly Discovered Work and its Background, Brepols, Turnhout, 2008: 63-80.

"Montezuma y la Conquista, una historia no resuelta [entrevista con Claudio Valdés Kuri]", El Universal, http://www.eluniversal.com.mx/cultura/63995.html. (Consultada en junio de 2014).

Moreno Mengíbar, Andrés, "España y la Ópera", Melómano http://orfeoed.com/melomano/2012/articulos/especiales/espana-y-la-opera/. (Consultada en junio de 2014).

Núñez Ronchi, Ana, "La reevaluación de las crónicas de Indias en la ópera The Indian Queen de Henry Purcell (1695)", Tesis de Doctorado de Arizona State University, 2003.

Núñez Ronchi, Ana, "Visión de la Conquista y de América en La Conquista de México (1668) de Fernando de Zárate", RILCE (Universidad de Navarra), 29/1 (2013): 389-414.

Núñez Ronchi, Ana. "De las crónicas de Indias a la ópera barroca: discursos y géneros". Actas del coloquio Empreintes / emprunts: entre forces d'innovation et forces de conformisation. 4-5 jun. 2012, Lorraine, Université de Lorraine, 2014: 111-127.

Orr, Bridget, Empire on the Restoration Stage: 1660-1714, Cambridge, Cambridge University Press, 2001.

Palm, Erwin W., "El indio como objeto del teatro: el teatro de la restauración inglesa y la ópera de Purcell", La imagen del indio en la Europa moderna, Sevilla, CSIC, 1990: 85-99. 
Pinnock, Andrew y Laurie, Margaret, "Preface”, Purcell: The Indian Queen, Londres, Novello, 1994: ix-xxix.

Price, Curtis A, Henry Purcell and the London Stage, Cambridge, Cambridge University Press, 1984.

Purcell, Henri, The Songs in the Indian Queen, Londres, Printed by J. Heptinstall, and are to be sold by John May [...] and for John Hudgbutt at Tho[mas] Daring's, 1695

Rameau, Jean-Philippe, Les Indes Galantes, París, Ballard, 1736.

Rameau, Jean-Philippe, Les Indes Galantes, Livret et avertissement, París, Erato Disques, 1994.

Rastier, François, Essais de sémiotique discursive, París, Mame, 1973.

Rastier, "La généalogie d'Aphrodite. Réalisme et représentation artistique", Littérature, 87 (París, 1992): 105-123.

Rastier, François, "Les fondations de la sémiotique et le problème du texte. Questions sur les prolégomènes à une théorie du langage de Louis Hjelmslev", Texto! Textes et cultures, III (París, 1997), http://www.revue-texto.net/1996-2007/Inedits/Rastier/Rastier_Fondations.html. (Consultada en junio de 2014).

Rastier, François, Arts et sciences du texte, París, PUF, 2000.

Rastier, François, "Prédication, actance et zones anthropiques", Revue Texto! Textes et cultures XII/3 (París, 2007), http://www.revue-texto.net/1996-2007/Inedits/Rastier/Rastier_Predication-actance.pdf. (Consultada en junio de 2014).

Rastier, François, "Objets et performances sémiotiques. L'objectivation critique dans les sciences de la culture", Texto! Textes et cultures, XV/4 (París, 2010), http:// www.revue-texto.net/docannexe/file/2734/rastier_namur.pdf. (Consultada en junio de 2014).

Rastier, François, "Linguistique interprétative et fondements sémiotiques de la traduction", Texto! Textes et cultures, XVI/1 (París, 2011), http://www.revue-texto. net/docannexe/file/2718/tatiana_topicos_2.pdf. (Consultada en junio de 2014).

Ruiz Ramón, Francisco, "Introducción”, Francisco Ruiz Ramón (ed.), América en el teatro clásico español, Pamplona, Eunsa, 1993: 11-74.

Smith, John H., y Dougald MacMillan, eds. The Works of John Dryden, vol. 9, H.T. Swedenberg et al. (Eds. Grales), Berkeley: University of California Press, 1966.

Solís y Rivadeneyra, Antonio de, Historia de la Conquista de México, Madrid, En la Impr. de Bernardo de Villa-Diego, 1684.

Steele, Colin, English Interpreters of the Iberian New World from Purchas to Stevens: A Bibliographical Study (1603-1726), Oxford, Dolphin Book, 1975. 
Swed, Mark, “An Outrageous 'Queen' and Court”, Reseña de The Indian Queen de Henry and Daniel Purcell, David Schweizer (dir.), Guillermo Gómez-Peña (adapt.), Long Beach Opera, 14 y 20 jun. 1998, Los Angeles Times Online 16 jun. 1998, http://articles.latimes.com/1998/jun/16/entertainment/ca-60260. (Consultada en junio de 2014).

Traduction oecuménique de la Bible, París, Editions du Cerf, 1975-1976.

Vickers, David, "Introducción", Alan Curtis (dir.), Motezuma, CD, Il complesso barocco, Deutsche Grammophon, 2006: 48-58.

Vivaldi, Antonio, Alan Curtis (dir.), Motezuma, Il complesso barocco, Deutsche Grammophon, 2006.

Voss, Steffen, "Die Partitur von Vivaldis Oper Motezuma (1733)", Studi vivaldiani, 4 (Florencia, 2004): 53-72.

Zárate, Fernando de, "La conquista de México", Vern G. Williamsen (ed.), Parte treinta de comedias de los mejores ingenios de España, Madrid, Morrás, 1668, 228-259. http://www.cervantesvirtual.com/obra/la-conquista-de-mexico/. (Consultada en junio de 2014).

Zavala, Huguette, América inventada. Fiestas y espectáculos en la europa de los siglos XVI al XX, Antonio Urrutia y María José Riquelme (trads.), [Madrid], Banco Santander de negocios, 1994.

Fecha de recepción: 13 de junio de 2012.

Fecha de aceptación: 18 de febrero de 2013.

\section{American Indians on the European Lyric Stage: From the Purcell Brothers (1695) to Carl Heinrich Graun (1755)}

In contrast to a supposedly critically-oriented Golden Age comedia, the first American-themed operas (the Purcells, Vivaldi, Rameau, Graun) have been interpreted as uncritical expressions of the so-called Black Legend. Nevertheless, this judgment ought to be tempered: on the one hand, the Spanish comedia is not as censorious as has been asserted; on the other hand, although these operas do praise American Indians, they do not systematically belittle the Spaniards. Finally, the notion itself of "Black Legend" should be questioned.

KeY Words: opera; American Indians; Black Legend; interpretative semantics. 